\title{
SpringerLink
}

Athens Authentication Point

\section{Recognized as:}

Australian National University (761-54-630)

AUST LNCS (358-28-113) G08 Universities Australia (647-04-468)

\section{Welcome!}

To use the personalized features of this site, please log in or register.

If you have forgotten your username or password, we can help.

\section{My Menu}

Marked Items

Alerts

Order History

Saved Items

All

Favorites
Book Chapter

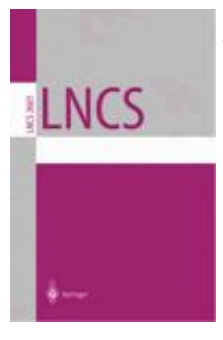

$\begin{array}{ll}\begin{array}{l}\text { A Fast Optimal Algorithm for } L_{2} \text { Triangulation } \\ \text { Book Series }\end{array} & \text { Lecture Notes in Computer Science } \\ \text { Publisher } & \text { Springer Berlin / Heidelberg } \\ \text { ISSN } & 0302-9743 \text { (Print) 1611-3349 (Online) } \\ \text { Volume } & \text { Volume 4844/2007 } \\ \text { Book } & \text { Computer Vision - ACCV 2007 } \\ \text { DOI } & 10.1007 / 978-3-540-76390-1 \\ \text { Copyright } & 2007 \\ \text { ISBN } & 978-3-540-76389-5 \\ \text { DOI } & 10.1007 / 978-3-540-76390-1 \_28 \\ \text { Pages } & 279-288 \\ \text { Subject Collection } & \text { Computer Science } \\ \text { SpringerLink Date } & \text { Wednesday, November 14, 2007 }\end{array}$

PDF (573.7 KB)

Find

Add to marked items

Add to shopping cart

Add to saved items

Recommend this chapter

\section{Looking for your books... ...on springer.com}

\section{Fangfang Lu ${ }^{1}$ and Richard Hartley ${ }^{1}$}

(1) Australian National University, Abstract

This paper presents a practical method for obtaining the global minimum to the least-squares $\left(L_{2}\right)$ triangulation problem.

Although optimal algorithms for the triangulation problem under $L$ $\infty$-norm have been given, finding an optimal solution to the $L 2$ triangulation problem is difficult. This is because the cost function under $L_{2}$-norm is not convex. Since there are no ideal techniques for initialization, traditional iterative methods that are sensitive to initialization may be trapped in local minima. A branch-and-bound algorithm was introduced in [1] for finding the optimal solution and it theoretically guarantees the global optimality within a chosen tolerance. However, this algorithm is complicated and too slow for large-scale use. In this paper, we propose a simpler branch-and-bound algorithm to approach the global estimate. Linear programming algorithms plus iterative techniques are all we need in implementing our method. Experiments on a large data set of 277,887 points show that it only takes on average $0.02 \mathrm{~s}$ for each triangulation problem.

\section{References}

1. Agarval, S., Chandraker, M., Kahl, F., Belongie, S., Kriegman, D.: Practical global optimization for multiview geometry. In: Proc. European Conference on Computer Vision (2005)

2. Hartley, R., Schaffalitzky, F.: $L \infty$ minimization in geometric reconstruction problems. In: Proc. IEEE Conference on Computer Vision and Pattern Recognition, Washington DC, pp. I-504-509 (2004)

3. Triggs, W., McLauchlan, P.F., Hartley, R.I., Fitzgibbon, A.: Bundle adjustment for structure from motion. In: Triggs, B., Zisserman, A., Szeliski, R. (eds.) Vision Algorithms: Theory and Practice. LNCS, vol. 1883, pp. 298-372. Springer, Heidelberg (2000)

Springerlink

\section{:}

$O$ Within this book Export this chapter Export this chapter as RIS | Text

\section{Ads by Google}

Quadratic Programming

Fast, Easy Portfolio Optimization Download Free Excel Models! www.solver.com

Algorithm Solutions Need a special Algorithm? ScienceOps has answers. www.ScienceOps.com

Production Optimization Production Planning \& Optimization Risk \& Decision Analysis www.petrovr.com

Need realistic 3D models?

Create 3D models from photos and export for web or $3 \mathrm{D}$ editing. www.3dsom.com

Solving polynomials? Get the solution now! Algebrator solves any polynomials problem. www.Algebra-Help.com/Polynomial 
4. Kahl, F.: Multiple view geometry and the $L \infty$-norm. In: Proc. International Conference on Computer Vision, pp. 1002-1009 (2005)

5. Hartley, R.I., Zisserman, A.: Multiple View Geometry in Computer Vision, 2nd edn. Cambridge University Press, Cambridge (2003)

6. Hartley, R.I., Sturm, P.: Triangulation. Computer Vision and Image Understanding 68(2), 146-157 (1997) cross $^{\text {ref }}$

7. Stewenius, H., Schaffalitzky, F., Nister, D.: How hard is 3view triangulation really. In: Proc. International Conference on Computer Vision, pp. 686-693 (2005)

8. Nocedal, J., Wright, S.J.: Numerical Optimization, 2nd edn. Oxford University Press, Oxford (2006)

MATH

9. Boyd, S., Vanderberghe, L.: Convex Optimization. Cambridge University Press, Cambridge (2004) MATH

10. Snavely, N., Seitz, S., Szeliski, R.: Photo tourism: Exploring photo collections in 3d. ACM Trans on Graphics 25(3), 835846 (2006) cross ${ }^{\text {ref }}$

Frequently asked questions | General information on journals and books | Send us your feedback | Impressum | Contact

(C) Springer. Part of Springer Science+Business Media

Privacy, Disclaimer, Terms and Conditions, (c) Copyright Information

Remote Address: $150.203 .208 .185 \cdot$ Server: mpweb17

HTTP User Agent: Mozilla/5.0 (Macintosh; U; Intel Mac OS X 10_4_11; en) AppleWebKit/525.13 (KHTML, like Gecko) Version/3.1 Safari/525.13 